\title{
Uso de medicamentos e suas potenciais interações com alimentos em idosos institucionalizados
}

\author{
Use of medicines and their potential interactions with foods in \\ institutionalized elderly individuals
}

\author{
Michele Sandri ${ }^{1} \bowtie$, Daiana Meggiolaro Gewehr ${ }^{2}$, Adriane Huth ${ }^{3}$, Angélica Cristiane Moreira ${ }^{4}$ \\ ${ }^{1}$ Curso de Nutrição da Universidade Regional do Noroeste do Estado do Rio Grande do Sul (Unijuí). ljuí, RS. \\ 2 Curso de Farmácia da Unijuí. ljuí, RS \\ ${ }^{3}$ Departamento de Ciências da Vida do Curso de Nutrição da Unijuí. ljuí, RS \\ ${ }^{4}$ Departamento de Ciências da Vida do Curso de Farmácia da Unijuí. ljuí, RS
}

\section{RESUMO}

Objetivos: Verificar o uso de medicamentos e identificar potenciais interações entre fármacos e alimentos/nutrientes em idosos institucionalizados. Métodos: Foi realizado um estudo transversal de abordagem quali-quantitativa. A coleta de dados ocorreu por meio da análise dos prontuários e prescrições médicas dos idosos residentes em uma instituição de longa permanência para idosos do Município de Ijuí-RS. A amostra, de conveniência, incluiu todos os idosos internados na instituição no período da coleta de dados: mês de setembro de 2015.

Resultados: Dos 30 idosos residentes na instituição, 29 utilizavam medicamentos de uso contínuo, com média de 8,72 $\pm 2,56$ medicamentos por idoso, totalizando 91 medicamentos distintos. Vinte e oito $(93,3 \%)$ idosos usavam cinco ou mais medicamentos, o que indica a existência de polifarmácia na grande maioria dos residentes na instituição. Os medicamentos mais utilizados atuam no sistema nervoso (35,07\%), seguidos dos que atuam no aparelho gastrointestinal ou no metabolismo (31,52\%) e no sistema cardiovascular (20,1\%). Constatou-se que 41 (45,05\%) medicamentos apresentavam potencial para interagir com alimentos, totalizando 142 interações. Potenciais interações foram identificadas

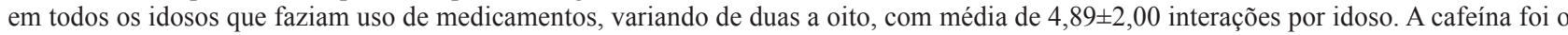
nutriente que esteve envolvido em mais interações, com potencial de interagir com nove $(21,95 \%)$ medicamentos distintos, sendo que destes, cinco (12,19\%) atuam no sistema nervoso.

Conclusões: Identificou-se alta frequência de polifarmácia pelos idosos institucionalizados, sendo os medicamentos de ação sobre o sistema nervoso os mais utilizados. Foram identificadas várias potenciais interações entre os fármacos e alguns tipos de alimentos/nutrientes presentes na alimentação.

DESCRITORES: instituição de longa permanência para idosos; instituições geriátricas de longa permanência; uso de medicamentos; interações alimento-droga; saúde do idoso.

\section{ABSTRACT}

Aims: To verify the use of medicines identifying their potential interactions with food/nutrients in institutionalized elderly individuals.

Methods: A qualitative and quantitative cross-sectional study was conducted. The data were collected through the analysis of medical charts and prescriptions of elderly individuals from a long-stay institution in Ijuí, Rio Grande do Sul State, Brazil. The convenience sample included all the elderly individuals institutionalized during the data collection period (September 2015).

Results: Of the 30 institutionalized elderly individuals, 29 were using continuous medication with a mean of $8.72 \pm 2.56$ medicines per individual, totaling 91 different drugs. Twenty-eight $(93.3 \%)$ individuals used five or more medicines, which indicates polypharmacy among most of the elderly residents. The most commonly used medicines were for the nervous system (35.07\%), digestive system and metabolism (31.52\%), and cardiovascular system (20.1\%). Forty-one (45.05\%) drugs could potentially interact with foods, totaling 142 interactions. Potential interactions were verified in all elderly individuals, ranging from two to eight, averaging $4.89 \pm 2.00$ interactions per elderly. Caffeine was involved in most interactions, potentially interacting with nine (21.95\%) different medications, five (12.19\%) of which acted on the nervous system.

Conclusions: There was a high frequency of polypharmacy among the institutionalized elderly and medicines to treat nervous system conditions were the most common ones. Several potential interactions between drugs and some types of foods/nutrients were verified.

KEY WORDS: homes for aged; geriatric long-term care facilities; drug utilization; food-drug interactions; elderly health.

Recebido: maio, 2016

Aceito: outubro, 2016

Publicado: novembro, 2016

Este artigo está licenciado sob forma de uma licença Creative Common 
Abreviaturas: ILPI, instituição de longa permanência para idosos; ATC, Anatomical Therapeutic Chemical Code; TGI, trato gastrointestinal; SNC, sistema nervoso central.

\section{INTRODUÇÃO}

A população de idosos nas últimas décadas vem aumentando gradualmente, sobretudo em consequência de ações de saúde pública e avanços na medicina e na tecnologia. $\mathrm{O}$ envelhecimento pode ser desencadeado de maneira natural, em que ocorre a diminuição progressiva da reserva funcional. Porém, na maioria dos casos ocorre uma condição patológica que acarreta danos e agravos à saúde, o que torna o idoso dependente [1].

A incidência de doenças origina-se do acúmulo de danos, ao longo da vida, oriundos sobretudo da interação entre fatores genéticos e hábitos não saudáveis, o que deixa os idosos mais propensos a desenvolver doenças crônicas [2,3]. Esses fatores associados a outros agravos à saúde são fatores que podem contribuir para a necessidade de transferência do idoso às instituições de longa permanência para idosos (ILPI) no intuito de que ele seja melhor cuidado [4].

Indivíduos que residem em ILPI tem alto risco de polifarmácia, por apresentarem muitas doenças limitantes, fragilidade e baixa funcionalidade [5]. A polifarmácia é caracterizada pelo uso de cinco ou mais medicamentos simultaneamente, e sua ocorrência é multifatorial, sendo que as doenças crônicas e as manifestações clínicas decorrentes do envelhecimento estão entre os principais fatores que influenciam essa situação [6]. Por outro lado, o uso de múltiplos fármacos predispõe à ocorrência de interações entre estes e os alimentos/nutrientes. Interação é definida como uma alteração dos processos cinéticos e/ou dinâmicos de um medicamento ou um nutriente, ou ainda, comprometimento do estado nutricional como resultado da administração de um medicamento.

Essas interações são complexas e difíceis de serem reconhecidas, o que pode ocasionar prejuízo na ação do medicamento e/ou do alimento. É necessário que tanto o alimento quanto o medicamento desempenhem sua ação desejada, sem comprometer a saúde do usuário. Desse modo, ao administrar medicamentos, é necessário observar dose e tempo de administração em relação à refeição, pois esses fatores poderão ser determinantes na ocorrência de interação entre os medicamentos e os alimentos [7-10].
Diante do exposto, é essencial conhecer as potenciais interações entre os fármacos e os alimentos/ nutrientes, para possibilitar um controle mais efetivo da administração dos medicamentos e da alimentação aos idosos, favorecendo a adoção de terapias mais eficazes. O presente estudo teve como objetivo verificar as potenciais interações entre fármacos e alimentos/nutrientes em idosos institucionalizados.

\section{MÉTODOS}

Foi realizado um estudo transversal em uma ILPI do município de Ijuí, localizado no interior do Rio Grande do Sul. A amostra, de conveniência, incluiu a totalidade dos idosos (idade acima de 60 anos) residentes na instituição na ocasião do estudo (setembro de 2015), e que aceitaram participar mediante assinatura do termo de consentimento livre e esclarecido, pelo idoso ou seu responsável legal. Nenhum critério de exclusão foi utilizado.

A coleta de dados ocorreu por meio da análise dos prontuários e prescrições médicas.

O projeto foi submetido à análise do Comitê de Ética em Pesquisa da Universidade Regional do Noroeste do Estado do Rio Grande do Sul e obteve aprovação sob parecer consubstanciado $\mathrm{n}$ ㅇ $1.185 .606 / 2015$. Foram respeitados os preceitos éticos contidos na Resolução no 466/2012 do Conselho Nacional de Saúde do Brasil [11].

Os medicamentos em uso pelos idosos foram classificados de acordo com o primeiro nível (grupo anatômico) e segundo nível (subgrupo terapêutico) da Classificação Anatômica Terapêutica Química (Anatomical Therapeutic Chemical Code) [12], proposta pela Organização Mundial da Saúde. Para verificar a ocorrência de potenciais interações entre os fármacos e os alimentos/nutrientes foi utilizada a base de dados Micromedex ${ }^{\circledR}$ [13] e demais referências $[8,14]$.

\section{RESULTADOS}

No período do estudo, 30 idosos eram residentes na ILPI e participaram da pesquisa. A média de idade dos participantes foi de 77,21 19,91 anos, com idade mínima de 60 e a máxima de 96 anos, sendo que $19(65,52 \%)$ eram do sexo feminino. Vinte e nove $(96,66 \%)$ moradores recebiam alimentação por via oral e uma $(3,33 \%)$ idosa utilizava sonda nasoentérica para alimentação com fórmula nutricional e administração de medicamentos. 
Utilização de medicamentos de uso contínuo foi constatada em $29(96,66 \%)$ dos participantes. A média foi de $8,72 \pm 2,56$ medicamentos por idoso. O número de prescrições totalizou 253, sendo que dessas, 91 medicamentos eram distintos e nas restantes ocorriam repetições do mesmo medicamento para mais de um idoso. A quantidade utilizada variou de quatro a 13 medicamentos, sendo que um $(3,3 \%)$ idoso utilizava quatro medicamentos e $28(93,3 \%)$ usavam cinco ou mais medicamentos, indicando a existência de polifarmácia na grande maioria dos participantes.
Quinze idosos $(51,72 \%)$ usavam nove ou mais medicamentos.

Os medicamentos mais utilizados foram os que atuam no sistema nervoso (35,07\%), seguidos dos que atuam no aparelho gastrointestinal e no metabolismo $(31,52 \%)$ e dos que atuam no sistema cardiovascular $(20,1 \%)$. O medicamento mais prescrito foi um suplemento vitamínico e mineral $(7,11 \%)$, utilizado por $18(62,06 \%)$ idosos, seguido de omeprazol (5,92\%), ácido acetilsalicílico $(4,74 \%)$ e paracetamol $(4,74 \%)$ (Tabela 1).

Tabela 1. Classificação por grupo terapêutico (ATC1) e subgrupo terapêutico (ATC2) dos fármacos utilizados por 30 idosos residentes em uma instituição de longa permanência para idosos. ljuí/RS, 2015.

\begin{tabular}{|c|c|c|}
\hline ATC1 - Grupo anatômico & ATC2 - Subgrupo terapêutico & n (\%) \\
\hline \multirow[t]{6}{*}{$\mathrm{N}$ - Sistema nervoso } & N02 - Analgésicos & $17(6,71)$ \\
\hline & N03 - Antiepilépticos & $13(5,13)$ \\
\hline & N04 - Antiparkinsonianos & $8(3,15)$ \\
\hline & N05 - Psicolépticos & $34(13,38)$ \\
\hline & N06 - Psicoanalépticos & $16(6,31)$ \\
\hline & N07 - Outros medicamentos do sistema nervoso & $1(0,39)$ \\
\hline Total & & $89(35,07)$ \\
\hline \multirow[t]{6}{*}{ A - Aparelho digestivo e metabolismo } & A02 - Antiácidos e medicamentos para o tratamento da úlcera péptica e flatulências & $18(7,09)$ \\
\hline & A03 - Medicamentos para disfunções funcionais gastrointestinais & $10(3,92)$ \\
\hline & A06 - Drogas para constipação & $12(4,73)$ \\
\hline & A10 - Medicamentos utilizados no diabetes & $10(3,95)$ \\
\hline & A11 - Vitaminas & $25(9,86)$ \\
\hline & A12 - Suplementos minerais & $5(1,97)$ \\
\hline Total & & $80(31,52)$ \\
\hline \multirow[t]{7}{*}{ C - Sistema cardiovascular } & C01 - Terapêutica cardíaca & $6(2,36)$ \\
\hline & C02 - Anti-hipertensivos & $1(0,39)$ \\
\hline & C03 - Diuréticos & $16(6,31)$ \\
\hline & C07 - Betabloqueadores & $6(2,36)$ \\
\hline & C08 - Bloqueadores dos canais de cálcio & $2(0,78)$ \\
\hline & C09 - Agentes que atuam no sistema renina-angiotensina & $10(3,95)$ \\
\hline & C10 - Antidislipidêmicos & $10(3,95)$ \\
\hline Total & & $51(20,10)$ \\
\hline \multirow[t]{2}{*}{ B - Sangue e órgãos hematopoiéticos } & B01 - Medicamentos antitrombóticos & $12(4,74)$ \\
\hline & B03 - Preparados antianêmicos & $2(0,78)$ \\
\hline Total & & $14(5,52)$ \\
\hline \multirow[t]{2}{*}{$\mathrm{R}$ - Aparelho respiratório } & R03 - Medicamentos para doença obstrutiva das vias aéreas & $5(1,95)$ \\
\hline & R06 - Anti-histamínicos para uso sistêmico & $4(1,56)$ \\
\hline Total & & $9(3,51)$ \\
\hline G - Aparelho gênito-urinário e hormônios sexuais & G04 - Medicamentos urológicos & $4(1,56)$ \\
\hline Total & & $4(1,56)$ \\
\hline \multirow[t]{2}{*}{ M - Sistema músculo-esquelético } & M01 - Anti-inflamatórios e antirreumáticos & $3(1,17)$ \\
\hline & M04 - Preparados antigotosos & $1(0,39)$ \\
\hline Total & & $4(1,56)$ \\
\hline H - Preparados hormonais sistêmicos & H03 - Terapêutica tireoidiana & $2(0,79)$ \\
\hline Total & & $2(0,79)$ \\
\hline S - Órgãos dos Sentidos & S01 - Produtos oftalmológicos & $1(0,39)$ \\
\hline Total & & $1(0,39)$ \\
\hline Número total de medicamentos & & $253(100)$ \\
\hline
\end{tabular}

ATC, Anatomical Therapeutic Chemical Code. 
Tabela 2. Potenciais interações entre fármacos e alimentos/nutrientes em 30 idosos residentes em uma instituição de longa permanência para idosos. ljuí/RS, 2015.

\begin{tabular}{|c|c|c|c|}
\hline Alimentos/nutrientes & Fármaco & Efeito da interação & n (\%) \\
\hline \multicolumn{4}{|l|}{ Sistema Nervoso } \\
\hline Alimentos, Sódio & Carbonato de lítio ${ }^{6,14,13}$ & $\begin{array}{l}\uparrow \text { da exposição do fármaco pelos alimentos } \\
\downarrow \text { do efeito terapêutico pelo excesso de sódio }\end{array}$ & $1(0,7)$ \\
\hline \multirow[t]{5}{*}{ Cafeína } & Clonazepam ${ }^{6,14,13}$ & $\downarrow$ do efeito desses fármacos pelo consumo de alimentos e bebidas cefeinadas & $6(4,2)$ \\
\hline & Clorpromazina $^{6,14}$ & & $7(4,9)$ \\
\hline & Amitriptilina $a^{6,14}$ & & $3(2,1)$ \\
\hline & Haloperido|6,14 & & $2(1,4)$ \\
\hline & Alprazolam $6,14,13$ & & $1(0,7)$ \\
\hline Proteínas & Levodopa $^{6,14,13}$ & $\downarrow$ da absorção do fármaco & $3(2,1)$ \\
\hline \multirow[t]{5}{*}{ Alimentos } & Paracetamol $\left.\right|^{6,13}$ & $\downarrow$ da absorção do fármaco e retardo do início de ação & $13(9,1)$ \\
\hline & Pramipexol $^{14}$ & & $1(0,7)$ \\
\hline & Zolpidem $^{14,13}$ & & $5(3,5)$ \\
\hline & Quetiapina $^{14}$ & da absorção e biodisponibilidade do fármaco & $1(0,7)$ \\
\hline & Carbamazepina ${ }^{1,2}$ & & $7(4,9)$ \\
\hline Total & & & $50(35,2)$ \\
\hline \multicolumn{4}{|l|}{ Sistema Digestivo e Metabólico } \\
\hline \multirow[t]{2}{*}{ Alimentos em geral } & Ácido Fólico ${ }^{6,14}$ & $\uparrow$ a biodisponibilidade do ácido fólico & $1(0,7)$ \\
\hline & Metformina ${ }^{6,14}$ & $\downarrow$ da absorção de vitamina $B_{12}$ e folato & $4(2,8)$ \\
\hline Leite & Bisacodil $6,14,13$ & Causa irritação gástrica ou duodenal & $2(1,4)$ \\
\hline Fibras, Oxalato e fitato & Cálcio $^{14}$ & $\downarrow$ da eficácia do fármaco & $5(3,5)$ \\
\hline Bebidas gaseificadas & Dimeticona e simeticona ${ }^{14}$ & $\uparrow$ da formação de gases estomacais & $3(2,1)$ \\
\hline Vitaminas e minerais & $\begin{array}{l}\text { Hidróxido de magnésio + } \\
\text { carbonato de cálcio }+ \\
\text { hidróxido de alumínio }\end{array}$ & $\begin{array}{l}\text { Inativa a tiamina e diminui a absorção de vitamina A, ácido fólico, potássio, } \\
\text { ferro, fosforo e cálcio }\end{array}$ & $1(0,7)$ \\
\hline Líquidos, Fibras & Leite de magnésia ${ }^{14}$ & Melhoram o efeito do fármaco & $1(0,7)$ \\
\hline Vitaminas e minerais & Óleo mineral ${ }^{6,14}$ & $\downarrow$ a absorção de água, vitaminas e minerais & $9(6,3)$ \\
\hline Ferro e vitamina $B_{12}$ & Omeprazol e pantoprazol ${ }^{14}$ & $\downarrow$ a absorção de ferro e vitamina $B_{12}$. Deve ser ingerido em jejum & $16(11,3)$ \\
\hline Vitaminas e cafeína & Ranitidina $^{6,14}$ & $\downarrow$ a absorção de vitamina $A, B_{1}$ e $B_{12}$. A cafeína diminui sua absorção & $1(0,7)$ \\
\hline Total & & & $43(30,2)$ \\
\hline \multicolumn{4}{|l|}{ Sistema Cardiovascular } \\
\hline \multirow{2}{*}{ Potássio } & Espironolactona $a^{6,14}$ & $\uparrow$ o risco de hipercalemia & $3(2,1)$ \\
\hline & Amilorida ${ }^{6,13}$ & & $1(0,7)$ \\
\hline Magnésio e cálcio & Captopril6,14,13 & $\downarrow$ na absorção e biodisponibilidade do fármaco & $2(1,4)$ \\
\hline Fibras e vitamina $D$ & Digoxina ${ }^{6,14,13}$ & $\begin{array}{l}\text { As fibras podem } \downarrow \text { a concentração e o pico das concentrações de digoxina. O } \\
\text { excesso de vitamina } D \text { pode } \uparrow \text { a toxicidade do fármaco. }\end{array}$ & $4(2,8)$ \\
\hline \multirow[t]{6}{*}{ Alimentos em geral } & Hidroclorotiazida ${ }^{6,14}$ & $\uparrow$ da absorção com alimentos que retardam o esvaziamento gástrico. & $6(4,2)$ \\
\hline & Furosemida ${ }^{6,14,13}$ & $\downarrow$ da eficácia do fármaco, principalmente alimentos ricos em sódio & $6(4,2)$ \\
\hline & Atenolol $\left.\right|^{6,14}$ & $\downarrow$ da absorção do fármaco & $3(2,1)$ \\
\hline & Ciprofibrato $^{6}$ & & $1(0,7)$ \\
\hline & Isordil ${ }^{14}$ & & $1(0,7)$ \\
\hline & Metropolo| ${ }^{6,14,13}$ & $\downarrow$ das concentrações do fármaco & $1(0,7)$ \\
\hline Proteínas e cafeína & Verapamil ${ }^{6,13}$ & $\begin{array}{l}\text { Proteínas } \uparrow \text { da absorção do fármaco } \\
\text { Cafeína pode } \uparrow \text { a estimulação do SNC }\end{array}$ & $1(0,7)$ \\
\hline Total & & & $29(20,4)$ \\
\hline \multicolumn{4}{|l|}{ Outros } \\
\hline \multirow[t]{5}{*}{ Alimentos em geral } & Ácido Acetilsalicílico ${ }^{6,14}$ & $\downarrow$ a absorção, porém ingestão de alimentos diminui os distúrbios do TG & $12(8,4)$ \\
\hline & Sulfato ferroso $\mathrm{O}^{14,13}$ & $\downarrow$ da absorção do fármaco & $1(0,7)$ \\
\hline & Tansulosina $^{14,13}$ & $\downarrow$ da biodisponibilidade do fármaco & $1(0,7)$ \\
\hline & Colchicina $^{6,14}$ & $\begin{array}{l}\text { O fármaco } \downarrow \text { a absorção de gordura, proteínas, sódio, potássio, fósforo e } \\
\text { vitamina } B_{12}\end{array}$ & $1(0,7)$ \\
\hline & Diclofenaco ${ }^{6,14}$ & $\downarrow$ a taxa de absorção & $1(0,7)$ \\
\hline Ferro & Levotiroxina sódica ${ }^{6,14,13}$ & $\downarrow$ da absorção do fármaco & $2(1,4)$ \\
\hline Proteínas, carboidratos e cafeína & Aminofilina ${ }^{6,13}$ & $\begin{array}{l}\text { Dieta hiperproteíca retarda a absorção dessa droga, já uma dieta hiperglicídica } \\
\text { acelera sua absorção. A cafeína } \uparrow \text { das concentraçôes desse fármaco }\end{array}$ & $1(0,7)$ \\
\hline Cálcio e cafeína & Prednisona $a^{6,14}$ & $\begin{array}{l}\text { Provoca perda de cálcio, deficiência de fosfato e vitamina D } \\
\text { A cafeina aumenta os desconfortos gastrointestinais. }\end{array}$ & $1(0,7)$ \\
\hline Total & & & $20(14,0)$ \\
\hline Total de interações distintas: 41 & & & $142(100)$ \\
\hline
\end{tabular}

Fontes: Reis NT'; Martins C, Saeki SL ${ }^{14}$; Micromedex (2015) ${ }^{13}$. 
As refeições realizadas pelos idosos eram fracionadas em seis por dia (desjejum, lanche da manhã, almoço, lanche da tarde, jantar e ceia). A alimentação de cada idoso era variada conforme sua comorbidade e preferência, sendo orientada pela nutricionista, responsável técnica no local. Em relação à concomitância da administração dos fármacos com os horários das refeições, os funcionários não levavam essa questão em consideração. Eles seguiam a prescrição médica com os devidos horários dos medicamentos, sendo que alguns coincidiam com o momento das refeições.

Em relação às potenciais interações entre fármacos e alimentos/nutrientes, constatou-se que 41 (45,05\%) medicamentos apresentavam potencial para interagir com alimentos, com frequência de 142 interações no total. Constatou-se que todos os idosos do estudo estavam sujeitos a pelo menos uma interação entre fármacos e alimentos/nutrientes. $\mathrm{O}$ número de possíveis interações variou de duas a oito, sendo a

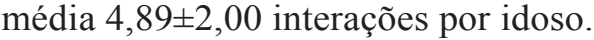

Do total das possíveis interações, 12 (29,27\%) eram com medicamentos que atuam sobre o sistema nervoso; $11(26,83 \%)$ com agentes cardiovasculares; $10(24,39 \%)$ com fármacos que atuam no sistema gastrointestinal; e oito $(19,5 \%)$ com fármacos que atuam sobre os demais sistemas. Em relação aos nutrientes e alimentos envolvidos nas interações com os fármacos estavam cafeína, carboidratos, proteínas, fibras, oxalato, fitato, vitaminas, minerais, leite e bebidas gaseificadas. A cafeína foi o nutriente que esteve envolvido em mais interações, com potencial de interagir com nove $(21,95 \%)$ medicamentos distintos, cinco destes $(12,19 \%)$ com ação no sistema nervoso (Tabela 2).

\section{DISCUSSÃO}

Cada ILPI apresenta características próprias, e o uso de medicamentos varia conforme cada instituição. Os idosos deste estudo apresentavam alta prevalência de polifarmácia, de forma que se poderia inferir que o risco de interações fármaco versus alimentos/nutrientes fosse alto, o que foi confirmado.

O sexo feminino foi o que apresentou predominância entre os residentes na instituição. Outros estudos realizados com idosos institucionalizados também constataram maior frequência do sexo feminino nesses espaços [15-17]. Em termos de quantidade de medicamentos utilizada, um aspecto importante a ser considerado é a faixa etária, pois à medida que a idade avança, aumentam os riscos da pessoa adoecer, de ter maior grau de dependência e, consequentemente, de utilizar mais medicamentos. Dessa forma, em instituições que abrigam idosos mais velhos, a média de uso de medicamentos pode ser maior $[7,16]$.

A taxa média de uso de medicamentos obtida nesse estudo foi semelhante à de um estudo realizado por Ribeiro et al. [18] em uma ILPI de Viamão/RS, com predomínio de idosos do sexo feminino e média de idade de 85 anos, obtiveram uma média de 8,1 medicamentos por pessoa. Já em outros estados do Brasil, a média de uso de medicamentos em ILPI foi menor. Fochat et al. [16], em estudo realizado com 122 idosos institucionalizados residentes em Minas Gerais, verificaram a média de seis medicamento por idoso. Enquanto Peixoto et al. [19], em estudo realizado no estado do Paraná com 73 idosos institucionalizados, obtiveram a média de 4,7 medicamentos por idoso.

A presença de múltiplas doenças também é um fator determinante para o uso de múltiplos fármacos [16]. Entre os idosos participantes deste estudo, a metade utilizava mais de nove medicamentos, e mais de 90\% alcançavam o conceito de uso de polifarmácia (cinco medicamentos ou mais); além disso, 45,05\% dos medicamentos prescritos apresentavam potencial de interagir com os nutrientes. $\mathrm{O}$ estudo de Peixoto et al. [19], obteve o total de 345 medicamentos em 166 receitas, sendo que $32 \%$ faziam algum tipo de interação com alimentos/nutrientes.

Quando são prescritos múltiplos fármacos aos idosos, há aumento do risco de deficiências nutricionais, pois podem ocorrer prejuízos na digestão, absorção, excreção e biodisponibilidade de muitos nutrientes. Desse modo, o conhecimento das questões relacionadas aos medicamentos e nutrientes é importante para planejar o tipo de cuidado necessário para evitar as deficiências nutricionais $[19,20]$.

Além disso, nos idosos as condições nutricionais estão associadas a mudanças provocadas pelos processos fisiológicos e patológicos. Dessa forma, os idosos são mais suscetíveis a apresentar problemas decorrentes das interações entre fármacos e alimentos/ nutrientes, já que a idade avançada predispõe a uma série de dificuldades nos processos fisiológicos (diminuição do funcionamento gastrointestinal, da produção de suco gástrico, das funções hepática e renal e, até, da ligação do fármaco com as proteínas séricas), podendo afetar a distribuição, excreção e absorção dos fármacos, alterando a resposta farmacológica esperada $[21,22]$.

A alta porcentagem de medicamentos que atuam no sistema nervoso sobre todos os medicamentos identificados neste estudo (35\%, sendo essa a classe mais 
utilizada), é semelhante às porcentagens encontradas em outros estudos com idosos institucionalizados, que também identificaram maior frequência de prescrição dessa classe de medicamentos: $38,8 \%$ [16] e $37,6 \%$ [19].

Os fármacos administrados por via oral apresentam como principal local de interação com alimentos/ nutrientes o trato gastrointestinal, já que ambos (medicamentos e nutrientes) necessitam passar pelo processo de absorção. A composição da dieta influencia o tempo de permanência dos fármacos no trato digestivo e, consequentemente, aumenta ou diminui a absorção dos mesmos [7]. No presente estudo, os medicamentos eram administrados em horários padronizados pela ILPI, sem que fossem levadas em conta as refeições, portanto, sem atentar para a ocorrência de possíveis interações entre fármacos e alimentos. Constatase a necessidade de aplicar os conhecimentos sobre interações fármacos/nutrientes ao planejamento e organização das ILPI.

O fracionamento das refeições em seis ou mais vezes ao dia justifica-se devido ao fato de que a própria senescência, e alguns agravos à saúde, podem provocar gastroparesia e saciedade precoce [7]. Dessa forma, é importante que os idosos ingiram alimentos em menores volumes e maior frequência, buscando atingir as necessidades calóricas e nutricionais. Esse fato acarreta ainda maior necessidade de atenção aos horários da medicação.

Há fármacos que devem ser ingeridos com alimentos para evitar o desconforto gastrointestinal, como clonazepam, alprazolam, carbonato de lítio, levodopa e carbamazepina [8]. Entretanto, devem ser lembradas possíveis interações, como no caso do carbonato de lítio, que pode ter sua concentração aumentada devido ao aumento da absorção na presença de alimentos [13].

Alguns fármacos (como paracetamol, zolpidem, atenolol, captopril, digoxina, sulfato ferroso e tansulosina), necessitam ser administrados longe das refeições, pois o alimento tem a capacidade de diminuir a sua absorção. Ao contrário, alguns fármacos (como carbamazepina, carbonato de lítio, ácido fólico, verapamil e hidroclorotiazida) devem ser ingeridos perto das refeições para que ocorra aumento na sua absorção $[8,13,19]$. Assim, o intervalo de tempo entre a administração do fármaco e a refeição é determinante na terapêutica. Esse cuidado deve ser tomado a fim de evitar ou reduzir problemas relacionados à má absorção tanto dos fármacos quanto dos nutrientes [19]. Salientase que o desenvolvimento de um programa de interação fármaco-nutriente envolvendo um grupo profissional multidisciplinar pode contribuir para a segurança e eficácia dos processos terapêuticos [10].

Cabem algumas considerações sobre alguns fármacos específicos de uso frequente pelos idosos. Entre os mais utilizados está o paracetamol, que quando ingerido juntamente com a alimentação, além de sofrer prejuízo em sua absorção, pode diminuir a absorção das vitaminas B1, B6, K e do ácido fólico [8].

A cafeína está entre os principais compostos com atividade biológica ingeridos pela população. Apresenta ação estimulante sobre o sistema nervoso central e o cardiovascular, atua como diurético e como relaxante muscular $[19,23]$. Encontra-se em maior concentração em alimentos ou bebidas como café, chá preto, erva-mate, chocolate e guaraná [24]. Verificaram-se cinco interações envolvendo a cafeína com a classe de medicamentos de ação no sistema nervoso. Por sua ação estimulante, a cafeína interfere principalmente com ansiolíticos e sedativos. Peixoto et al. [19] também verificaram que a interação mais frequente $(32 \%)$ envolveu a cafeína.

Em relação às potenciais interações entre alimentos e fármacos que agem sobre o sistema gastrointestinal, pode-se destacar a frequente utilização de laxantes, como o bisacodil e o óleo mineral, que quando administrados perto das refeições podem ocasionar deficiência de vitaminas lipossolúveis $(\mathrm{A}, \mathrm{D}, \mathrm{E}$ e K) e reduzir a absorção de glicose, proteína, sódio, potássio e cálcio [7]. Sendo assim, é recomendado que a administração desses fármacos seja feita longe das refeições, em geral de manhã cedo ou antes de dormir [21].

Os agentes antiulcerosos, como o omeprazol e a ranitidina, prejudicam a absorção da vitamina B12. Portanto, esses fármacos não devem ser administrados junto às refeições que contenham os principais alimentos fontes dessa vitamina (carne, frango e leite) [21].

Estudos realizados em idosos não institucionalizados identificaram como mais frequente o consumo de fármacos que atuam sobre o aparelho cardiovascular [5,25]. Todavia, no presente estudo, assim como em outros que incluíram idosos institucionalizados [16,19], o consumo desses fármacos foi menos frequente do que o daqueles que atuam sobre o sistema nervoso.

Em relação aos medicamentos que atuam sobre o sistema cardiovascular, os diuréticos foram os mais utilizados pelos idosos no presente estudo, sendo os mais frequentes a furosemida e a hidroclorotiazida. $\mathrm{O}$ uso prolongado ou o consumo de altas doses desses medicamentos podem ocasionar desidratação e depleção de minerais como potássio, magnésio e zinco [7]. 
Por outro lado, alimentos ricos em sódio diminuem a concentração e eficácia da furosemida [14], portanto é necessário monitorar a ocorrência dessas possíveis interações.

Pode-se concluir, com este estudo, que houve alta frequência de polifarmácia pelos idosos institucionalizados, sendo os medicamentos de ação sobre o sistema nervoso os mais utilizados. Foram identificadas várias potenciais interações entre os fármacos e alguns tipos de alimentos/nutrientes frequentes na alimentação. Nesse sentido, a divulgação de estudos como o presente é fundamental para enfatizar e disseminar a importância do cuidado com o tipo de alimentação e os medicamentos utilizados em instituições para idosos, visto que as interações podem interferir na saúde e trazer prejuízos tanto ao efeito terapêutico desejado quanto ao estado nutricional dos indivíduos expostos.

\section{NOTAS}

\section{Apoio Financeiro}

Serviço de Amparo e Bem Estar da Velhice, Universidade Regional do Noroeste do Estado do Rio Grande do Sul, Departamento de Ciências da Vida.

Agradecimento

À instituição de longa permanência para idosos pela disponibilização das informações, o que tornou possível a realização deste trabalho.

Declaração de conflitos de interesse

Os autores declaram não haver conflitos de interesse relevantes ao conteúdo deste estudo.

\section{REFERÊNCIAS}

1. Ministério da Saúde (BR). Secretaria de Atenção à Saúde. Departamento de Atenção Básica. Envelhecimento e saúde da pessoa idosa. Brasília-DF: Ministério da saúde; 2007. 192 p.

2. Moraes EN. Atenção à saúde do idoso: aspectos conceituais. Brasília-DF: OPAS; 2012. 98 p.

3. Leite LEDA, Resende TDL, Nogueira GM, Cruz IBM Da, Schneider RH, Gottlieb MGV. Envelhecimento, estresse oxidativo e sarcopenia: uma abordagem sistêmica. Rev Bras Geriatr Gerontol. 2012;15(2):365-80. http://dx.doi.org/10.1590/S1809-98232012000200018

4. Terassi M, Rissardo LK, Peixoto JS, Salci MA, Carreira L. Prevalência do uso de medicamentos em idosos institucionalizados: um estudo descritivo. Online Braz J Nurs. 2012;11(1):26-39. http://dx.doi.org/10.5935/1676-4285.20120004

5. Carvalho MFC, Romano-Lieber NS, Bergsten-Mendes G, Ribeiro SRSE, Lebrão ML, Duarte YA de O. Polifarmácia entre idosos do Município de São Paulo - Estudo SABE. Rev Bras Epidemiol. 2012;15(4):817-27. http://dx.doi.org/10.1590/S1415-790X2012000400013

6. Secoli SR. Polifarmácia: interações e reações adversas no uso de medicamentos por idosos. Rev Bras Enferm. 2010;63(1):136-40. http:// dx.doi.org/10.1590/S0034-71672010000100023

7. Moura MRL, Reyes FGR. Interação fármaco-nutriente: uma revisão. Rev Nutr. 2002;15(2):223-38. http://dx.doi.org/10.1590/S141552732002000200011

8. Reis NT. Nutrição Clínica: Interações. Rio de Janeiro: Editora Rubio; 2004. 604 p.

9. Carvalho AMR, Oliveira DC, Neto JEDH, Martins BCC, Vieira VMDSF, Silva LIMM da, et al. Análise da prescrição de pacientes utilizando sonda enteral em um Hospital Universitário do Ceará. R Bras Farm Hosp Serv Saúde. 2010;1(1):17-21.

10. Heldt T, Loss SH. Interação fármaco-nutriente em unidade de terapia intensiva: revisão da literatura e recomendações atuais. Rev Bras Ter Intensiva. 2013;25(2):162-7. http://dx.doi.org/10.5935/0103-507X.20130028

11. Ministério da Saúde (BR); Conselho Nacional de Saúde. Resolução nº 466, de 12 de dezembro de 2012. Brasília; 2012.

12. WHO Collaborating Centre for Drug Statistics Methodology. Guidelines for ATC classification and DDD assignment 2014. Oslo: Norwegian Institute of Public Health; 2015.

13. Micromedex ${ }^{\circledR}$ Healthcare Series [Internet database]. Greenwood Village, CO: Thomson Micromedex; 2015 [updated periodically; cited 2015 Out 15]. Available from http://www.thomsonhc.com/

14. Martins C, Saeki SL. Interações Fármaco X nutriente. 3ae ed. Curitiba: Instituto Cristina Martins; 2013. 238 p.

15. Gonçalves LH, Silva AH, Mazo GZ, Benedetti TR, dos Santos SM, Marques S, Rodrigues RA, Portella MR, Scortegagna HM, Santos SS, Pelzer MT, Souza Ados S, Meira EC, Sena EL, Creutzberg M, Resende TL. O idoso institucionalizado: avaliação da capacidade funcional e aptidão física. Cad Saude Publica. 2010 Sept; 26(9):1738-46. http://dx.doi.org/10.1590/S0102-311X2010000900007

16. Fochat RC, Oliveira Horsth RB de, Sette MS, Barbosa Raposo NR, Chicourel EL. Perfil de utilização de medicamentos por idosos frágeis institucionalizados na Zona da Mata Mineira, Brasil. Rev Cienc Farm Básica Apl. 2012;33(3):447-54.

17. Cruz HL, Cruz MFK, Andrade RA, Bodevan EC, Araújo LU, Santos DF. Caracterização do uso de medicamentos entre idosos de uma unidade do Programa Saúde da Família. Cad Saúde Pública. 2014;24(7):1545-55. http://dx.doi.org/10.1590/S0102-311X2008000700009 
18. Ribeiro NP, Mascarenhas R, Mascarenhas MA, Gutierrez LLP. Polifarmácia utilizada por idosos residentes em instituições de longa permanência do município de Viamão/RS. Ciênc Mov. 2013;65-74.

19. Peixoto JS, Salci MA, Radovanovic CAT, Salci TP, Torres MM, Carreira L. Riscos da interação droga-nutriente em idosos de instituição de longa permanência. Rev Gaúcha Enferm. 2012;33(3):156-64. http://dx.doi.org/10.1590/S1983-14472012000300021

20. Silva R, Schmidt O, Silva S. Polifarmácia em geriatria. Rev AMRIGS. 2012;56(2):164-74.

21. Lopes EM, Carvalho RBN, Freitas RM. Análise das possíveis interações entre medicamentos e alimento/nutrientes em pacientes hospitalizados. Einstein. 2010; 8(3 Pt 1):298-302. http://dx.doi.org/10.1590/S1679-45082010AO1672

22. Leibovitch ER, Deamer RL, Sanderson LA. Food-drug interactions: Careful drug selection and patient counseling can reduce the risk in older patients. Geriatrics. 2004 Mar;59(3):19-22, 32-3.

23. Maria CAB, Moreira RFA. Cafeína: revisão sobre métodos de análise. Quim Nova. 2007;30(1):99-105. http://dx.doi.org/10.1590/S010040422007000100021

24. Brenelli E, Cristina S. A extração de cafeína em bebidas estimulantes - uma nova abordagem para um experimento clássico em química orgânica. Quim Nova. 2003;26(1):136-8. http://dx.doi.org/10.1590/S0100-40422003000100023

25. Bueno CS, Oliveira KR, Berlezi EM, Eickhoff HM, Dallepiane LB, Mafalda A. Uso de fármacos e risco de interações medicamentosas em idosas acompanhadas pelo Programa de Atenção ao Idoso na área urbana de um município do Rio Grande do Sul. RBCEH. 2012;9(1): $119-28$.

26. Faria MQ, Franceschini SCC, Ribeiro AQ. Estado nutricional e uso de medicamentos por idosos. Lat Am J Pharm. 2010;29(1): $127-31$. 